\title{
Using mixed methods to design a web-based behavioural intervention to reduce transmission of colds and flu
}

\author{
L. Yardley ${ }^{1}$, S. Miller ${ }^{*}$, E. Teasdale ${ }^{1}$ and P. Little ${ }^{2}$ on behalf of the PRIMIT team. \\ ${ }^{1}$ School of Psychology and ${ }^{2}$ School of Medicine, University of Southampton, Southampton \\ SO17 1BG, UK \\ * Correspondence to S.Miller. Email: sm2t07@soton.ac.uk \\ PRIMIT team also includes Richard Hobbs and Bill Carmen.
}

Running head: Developing a web-based intervention using mixed methods

Keywords: health promotion; internet; human influenza; qualitative research; questionnaires Word count (excluding abstract, tables, references): 4333 


\begin{abstract}
This mixed methods study informed the development of our web-based intervention to reduce the risk of transmission of respiratory infections. Two qualitative studies examined 28 users' responses to website materials, and elicited additional beliefs. A questionnaire study ( $N$ =129) tested key intervention assumptions: that hand-washing is considered the most feasible preventive behaviour; behavioural determinants were related to behavioural intentions; beliefs related to hand-washing intentions and behaviour. Thematic analysis of the qualitative data revealed important issues, such as the belief that catching minor infections is beneficial to the immune system. The questionnaire results confirmed our assumptions, including handwashing as the appropriate target behaviour.
\end{abstract}




\section{Introduction}

Colds and seasonal flu represent a common and persistent burden for individuals, medical services and society, resulting in reduced quality of life, absence from work, vulnerability to more serious illness and increased demand for medical services (Leaker, 2008; Madjid, Naghavi, Litovsky \& Casscells, 2003; Woodhead, Macfarlane, Mccracken, Rose \& Finch, 1987; Meier, Jick, Derby, Vasilakis, \& Jick, 1998). Research suggests that adoption of preventive measures such as hand-washing, mask-wearing and social isolation of the infected person may be an effective means of reducing transmission of respiratory infections, including colds and flu (Jefferson, Foxlee, Del Mar, Dooley, Ferroni, Hewak et al., 2008; Rabie \& Curtis, 2006; Cowling, Chan, Fang, Cheng, Fung, Wai W et al., 2009). These behaviours could become particularly important in the context of influenza pandemics Bell, Nicoll, Fukuda, Horby, Monto, Hayden et al.,2006), when there is an increased risk that normal socio-economic functioning could be disrupted and medical services overstretched. Slowing the spread of infection could help maintain the viability of health and other public services and permit the development of vaccine protection. However, more research is needed to optimise the design of interventions for promoting preventive behaviour, since adherence to recommendations is often not good (Jefferson et al., 2008; Rabie \& Curtis, 2006; Cowling et al., 2009). The aim of the research reported below was not only to inform the development of our own web-based intervention but also to provide an evidence-base for the development of other interventions to promote behaviours likely to reduce transmission of respiratory infections.

When planning our intervention, three potentially relevant sources of evidence about factors likely to influence our target preventive behaviours were reviewed. Since intervention development and testing began before the onset of the 2009 influenza pandemic, these sources were research on factors influencing hygienic behaviour in general, studies of 
preventive behaviour in SARS and studies of intended preventive behaviour in the event of an outbreak of pandemic flu.

Research on hand-washing in developing countries suggests that the primary motivation is seldom a rational intention to prevent disease; habitual, emotional and cultural influences appear to dominate handwashing behaviour, which is prompted by the desire for social acceptance and status, and emotions such as disgust and fear (Curtis, Danquah \& Aunger, 2009). Interestingly, a very similar pattern of motivations emerged in an Australian study of community members and nurses, in which hand-washing was cued by perceptions of obvious contamination and predicted by anticipated peer behaviour and pressure from doctors and administrators (Whitby, McLaws \& Ross, 2006). Surveys of responses to the SARS pandemic indicated that perceived risk and anxiety about infection as well as belief in the efficacy of preventive measures could motivate increased adherence to behaviours such as hand-washing and mask-wearing (Lau, Yang, Tsui \& Kim, 2003; Leung, Ho, Chan, Ho, Bacon-Shone, Choy et al., 2005; Tang \& Wong, 2004). Social norms, social pressure and understanding of the role of hygiene in prevention may also have been important (Lau et al.,2003; Cava, Fay, Beanlands, McCay \& Wignall, 2005; Slaughter, Keselman, Kushniruk \& Patel, 2005). Similar factors predicted reported intentions to adopt preventive behaviour in the event of pandemic flu among Hong Kong residents (Lau, Kim, Tsui \& Griffiths, 2007). However, there were marked variations in preventive behaviours in response to SARS in different communities and at different time-points in the epidemic (Lau et al., 2003; Leung, Quah, Ho, Hedley, Lee \& Lam, 2004), and so it is problematic to try to extrapolate directly from one context to another. To inform our intervention development we therefore undertook a series of qualitative and quantitative studies of attitudes towards preventive behaviours to reduce the risk of transmission of seasonal and pandemic flu in the UK. 


\section{Using mixed methods for intervention development}

The internet could provide a suitable medium for promoting behaviours to reduce transmission of pandemics, since online interventions can be delivered quickly and costeffectively to a large section of the population. To our knowledge, only one previous study has investigated the use of an online intervention to promote prevention of flu transmission (Bourgeois, Simons, Olson, Brownstein \& Mandl, 2008). Tailored messages about hand and cough-sneeze hygiene sent to participants resulted in positive trends in behaviour, although no significant effects were obtained in a small sample. The aim of the studies reported here was to inform the development of a web-based intervention to promote behaviours that might reduce the risk of cold and flu transmission between household members.

Conforming to best practice in intervention development Craig, Dieppe, Macintyre, Michie, Nazareth \& Petticrew, 2008; Gittelsohn, Steckler, Johnson, Pratt, Grieser, Piclcrel et al., 2006), we combined the use of theory and primary research to identify the factors likely to influence behaviour and determine how these might be addressed effectively by the intervention. The framework for intervention development that we employed was the PRECEDE-PROCEED model (Green \& Kreuter, 1999). The model recommends that developers first assess which behaviours are most likely to effect the desired clinical outcome and then determine which of these are most feasible to implement and acceptable to the target population. Our clinical team selected as potentially efficacious behaviours that might be suitable for non-pandemic as well as pandemic contexts: frequent hand-washing; social distancing (keeping at least a metre from infected people); and cough-sneeze hygiene (as promoted in the UK flu pandemic campaign, i.e. using a tissue to catch coughs and sneezes and disposing of it quickly in a bin). We then carried out a focus group study, which revealed that social distancing from family members was considered too uncaring, while doubts were expressed about the feasibility of consistently catching a cough or sneeze in a tissue and 
disposing of it while engaged in everyday activities (Morrison \& Yardley, 2009). Since hand-washing emerged as the most feasible and acceptable behaviour and also had the strongest evidence for efficacy, our prototype intervention focused principally on promoting frequent hand-washing.

The next steps in the PRECEDE-PROCEED model are to identify the psychological and environmental 'behavioural determinants' influencing performance of the target behaviour, develop intervention components that will influence these behavioural determinants, and pilot the intervention components and outcomes measures (Green \& Kreuter, 1999). We chose the Theory of Planned Behaviour (TPB; Ajzen, 1991) as the main framework for identifying the behavioural determinants, as it predicts health-related behaviour and can be applied in a wide variety of contexts (Godin \& Kok, 1996; Sutton, 2002; Webb \& Sheeran, 2006). The model proposes that behaviour is influenced by intentions, which are in turn influenced by attitudes towards the behaviour, subjective norms (perceptions of what behaviour others would approve of), and perceived behavioural control (i.e. feasibility of carrying out the behaviour). We applied the model to developing the intervention by creating messages that would lead participants to perceive hand-washing as necessary and effective (behavioural beliefs), socially desirable (normative beliefs) and easy to do (control beliefs).

The studies described below were carried out in order to check that our prototype intervention was targeting the appropriate behaviour and relevant determinants of behaviour. A mixed methods approach enabled us to obtain complementary information. Qualitative 'think aloud' methods are commonly employed in website development to elicit and observe reactions to using a website (Cotton \& Gresty, 2006; van den Haak, de Jong \& Schellens, 2007). We carried out 'think aloud' sessions to examine reactions to draft paper-based website materials and later to test the prototype website itself. These were combined with 
interviews based on the Theory of Planned Behaviour, to elicit salient behavioural, normative and control beliefs. This qualitative research allowed us to analyse in detail the ways in which different users responded to our messages, and to elicit any additional beliefs which our intervention might need to address.

The questionnaire study was used to test the assumptions on which our intervention was based. First we tested the hypothesis emerging from our focus group study that handwashing would be viewed as the most feasible preventive behaviour. We also used the questionnaire study to test whether, as expected, the Theory of Planned Behaviour constructs were related to behavioural intentions. Finally, we tested our assumption that the specific beliefs about hand-washing we had identified from the literature and qualitative research and addressed in our intervention would be related to hand-washing intentions and behaviour.

\section{Methods}

Ethical approval was obtained for all three studies from the National Research Ethics Service and the University of Southampton School of Psychology Ethics Committee. Invitation letters and advertisements for all studies indicated that participants must be adults living with at least one person (so that infection transmission within the household was possible).

\section{Qualitative studies}

Participants and procedure

The study of responses to the paper-based materials took place between December 2008 and January 2009, before the onset of the 2009 flu pandemic. Interviewees were recruited by advertisements placed around the University campus offering $£ 6.00$ reimbursement for their time. We purposively invited participants who felt that they (or someone they lived with) were at risk from catching colds or flu to simulate the heightened perceived risk we 
anticipated during a pandemic. Three men and ten women aged 20 to 69 were interviewed by the second author either in their own home or at the University. After giving written informed consent, participants were shown the paper-based materials in the sequence they would view them as web-pages and were asked to 'think aloud', i.e. to give their immediate reactions to each page. Following the think aloud session participants were asked: how they felt about using the proposed website materials and what would be the good and bad aspects of following the intervention advice (behavioural beliefs); what they thought others in their household would think of them following the advice (normative beliefs) and what would make it easier or harder to follow the advice (control beliefs). Interviews typically lasted an hour and were tape-recorded and fully transcribed.

The study of responses to the prototype website was carried out by the third author and a postgraduate student between August and September 2009, during the flu pandemic. To obtain a sample from our target primary care population, postal invitations to help with the development of our intervention by completing interviews or questionnaires were sent to 900 patients registered with three general practices, including areas of higher and lower socio-economic deprivation. Due to time constraints we arranged interviews with the first 15 people who agreed to participate; five men and ten women aged 41 to 71 . The procedure was the same as in the first qualitative study.

\section{Analysis}

An inductive thematic analysis was used to categorise the data (Joffe \& Yardley, 2004; Braun \& Clarke, 2006). The third author developed a coding manual and coded the data using manifest coding categories that were closely grounded in the text. The coding categories were organised into a hierarchy of categories and sub-categories, and final coding of all text segments and category labels was agreed with the first author. Interpretation of the coded 
text included consideration of whether statements were made spontaneously or in response to paper-based or web-based intervention materials.

\section{Questionnaire study}

Participants and procedure

A further 1,500 patients registered with the three general practices sampled previously plus another one were invited to complete a questionnaire (as above). Positive respondents from these and the 900 previous invitations were asked to complete the survey between August and October 2009, during the flu pandemic. Based on preferences stated on their consent form, 157 were emailed a link to the online survey and 63 were sent paper copies by post.

A total of 176 people completed the survey, but 6 (3.4\%) were excluded as they had already had swine flu and might therefore have different attitudes regarding preventive behaviour in the future. A further 41 (22.9\%) were excluded because they failed to complete measures of intention for all four behaviours. The 129 participants included in the analysis comprised 51 men and 75 women (3 did not give their gender) with a mean age of 53.89 (s.d. 13.61, range 19 to 82). Of the 126 who provided demographic data, 120 (95.2\%) lived with at least one family member aged 16 or over, 27 (21.4\%) were living with one or more children under 16 years of age, and 7 (5.6\%) were living with one or more unrelated adults.

\section{Measures}

Attitudes were measured by 7 point bipolar semantic differential scales, and past behaviour on a 5 point scale. All other items were scored from 1 (agree strongly) to 7 (disagree strongly). Summed sub-scale scores were divided by the number of items in the scale. Questions about each behaviour were prefaced by a precise definition of the behaviour, as follows: 'wash my hands at least 10 times a day with soap or antibacterial gel'; use a clean 
tissue every time I cough or sneeze and then immediately put it in a bin; always wear a mask when I have a virus, or when I am close to someone who may have a virus'; always keep a distance of 1 metre/3 feet away from someone who may have a virus, or from other people when I may have a virus’.

For each behaviour, we asked about frequency of current behaviour and then assessed behavioural beliefs. Two items formed a direct measure of instrumental attitude (useless/useful, necessary/unnecessary), and two measured affective attitude, i.e. how the behaviour would make the respondent feel (worried/confident, sensible/foolish). Two items asking about 'people whose opinions matter to me' and 'people I live with' assessed subjective norms (peer approval of the behaviour). Two items asking about perceived behaviour of these two peer groups assessed descriptive norms (Rivis \& Sheeran, 2003). After evaluating control beliefs, perceived behavioural control was assessed by two items, measuring the self-efficacy ('I am confident that I could) and perceived control ('it will be possible for me') dimensions, preceded by 'If I wanted to' to hold motivation constant (Ajzen, 2002; Rhodes, Blanchard \& Matheson, 2006). Intention to carry out the behaviour was assessed by two items asking if the respondent intended and would try to carry out the behaviour during the current pandemic.

Risk was assessed by two items assessing perceived likelihood of catching pandemic flu if no preventative action was taken, two items assessing perceived severity of the consequences of flu for health and and two item assessing anxiety about catching flu (Brewer, Chapman, Gibbons, Gerrard, McCaul \& Weinstein, 2007; Leppin \& Aro, 2009). We also assessed age, gender, household membership, and whether the respondent believed they had had swine flu.

\section{Analysis}


Missing values on single scale items were replaced with the sample mean for that item. We carried out four repeated measures ANOVAs (followed up by univariate tests and paired $t$ tests) to compare intentions and behavioural determinants of intentions for each behaviour. Bivariate Pearson's correlations were employed to examine the relationship of the TPB behavioural determinants to intentions for each behaviour, and multiple regressions to examine their combined contribution to variance in intentions. Current behaviour was entered on the first step of each regression, to focus the analysis on prediction of increased preventive behaviour (analyses of past behaviour were omitted for mask-wearing as only 7 people had ever worn a mask). The core TPB constructs were entered on the next step; attitudes (instrumental and affective), subjective norms and perceived behavioural control. Stepwise regression was employed on the last step to determine whether descriptive norms or any of the three risk measures explained further variance in intentions. The relationship of behavioural and control beliefs to hand-washing intentions and behaviour was assessed by bivariate Pearson's correlations.

\section{Results}

\section{INSERT HERE Table I}

\section{Qualitative studies}

The content of the qualitative data is summarised in Table I; the discussion below focuses on findings relevant to intervention development.

A powerful stereotype of how respiratory infections are transmitted was that they are principally airborne (often circulated by air-conditioning) and mainly caught in public places from conspicuously symptomatic people: 
'I think the biggest risk is other people. You have to stay away from people who catch colds, you cannot stay very close to them. It can be transmitted through air, especially in the bus, in the public area.' (P11 study 1; young woman)

'I've always thought that on an aeroplane, because they re-circulate the air, there's a chance that viruses and things are perhaps recycled through the air or ventilation system.’ (P7 study 2; older man)

Although some people knew of transmission by hand via infected surfaces, many were surprised to learn of the importance of this transmission route (though awareness appeared greater following the pandemic onset):

'Oh, I really didn't know this, this is very interesting, like you go somewhere in public and you touch something that has germs and then you touch your face and you don't really realise and that's how you get germs.' (P3 study 1 ; young woman)

While a range of negative effects of catching cold were mentioned, many people were fatalistic about the inevitability of infection and felt that catching colds was not a serious problem. Indeed, many believed that catching minor infections was useful for acquiring immunity and strengthening the immune system.

'I do think for minor viruses if you are too careful on minor viruses you will have a more sterile atmosphere and your immune system isn't as strong.' (P2, study 1; older woman) 
'We have a Chinese saying, like if you catch some kind of cold or flu, not a very serious one, just like a sneezing nose, something like that, from time to time, actually people in that kind of case may have less chance to get a very serious sickness.' ( $P 12$, study 1 ; young woman)

Hand-washing was generally regarded as a 'common-sense’ practice ingrained from childhood, rather than an intentional method of protection from infection. Most people felt they were already washing their hands at least 6 times a day (our initial target behaviour).

'I already do the necessary, like washing my hands after the loo, which is, well, common practice and basic hygiene and being aware of it.' (P8, study 1; younger woman)

Common perceived barriers to more frequent hand-washing included getting dry hands, and the difficulty of access to hygienic facilities outside the home. Hand-gel was seen as a potentially useful solution to cleansing outside the home, but not as an alternative to handwashing as it would not remove dirt.

Social concerns appeared to be a major potential barrier to adopting strict hygiene measures. Many hypothetical and some real examples were given of how taking hygienic precautions could give offence; for example, avoiding physical contact or eating in company unless hands were clean was considered socially unacceptable. A common fear was that scrupulous hand-washing could be viewed as - or might actually be - obsessive behaviour. 
'There is that sort of social element to it, feeling a bit awkward about it - "Have you got any hand-gel with you before I have that apple you're offering me on a picnic?’’ (P2, Study 2; older man)

'If I'd said [when meeting friends for lunch] ah, before we touch any food we must all go and wash our hands, I'm not sure what everybody's reaction would've been ... I think people would've looked at me as if I'm slightly mad.' (P9, study 2; older woman)

I did think about buying some [hand-gel] with all this swine flu stuff for when we go out and about. I haven't because I think that it just borders on the kind of obsessive.' (P13, study 2; mid-life woman)

\section{Questionnaire study}

Mean scores for measures of intentions and behavioural determinants for each behaviour are shown in Table II. As predicted, perceived behavioural control scores were substantially higher for hand-washing than for any of the other behaviours, including cough-sneeze hygiene, indicating that hand-washing was considered much the most feasible behaviour. Mask wearing and social distancing also had lower scores than hand-washing for attitudes and norms. However, intentions towards hand-washing and cough-sneeze hygiene did not differ significantly, and scores for attitudes and norms were higher for cough-sneeze hygiene than for hand-washing. This suggests that cough-sneeze hygiene is considered the most desirable behaviour, despite doubts about its practicability.

\section{INSERT HERE Table II}

As predicted by theory, intentions for each behaviour were strongly and significantly correlated with attitudes, subjective norms and perceived behavioural control for that 
behaviour (see Table III). The one exception was a weaker correlation between intentions and perceived behavioural control for hand-washing. The strongest associations were with subjective norms. Descriptive norms were also strongly correlated with cough-sneeze hygiene and social distancing, but less strongly related to hand-washing and mask-wearing. Measures of flu risk tended to have weaker relationships to intentions.

INSERT HERE Table III

Past behaviour was also strongly related to intentions. Just over half the sample $(\mathrm{n}=$ 67; 52.0\%) scored at least 4 out of 5, indicating that they reported currently hand-washing more than 6 times a day, and half of these reported already meeting the target of washing their hands at least 10 times a day $(n=34 ; 26.4 \%)$. A further 42 people $(32.6 \%)$ reported hand-washing 5 to 6 times a day, and 20 people (15.5\%) reported hand-washing 3 to 4 times a day. Reported rates of adherence to cough-hygiene measures were much lower; the median score was 2 out of 5, with 68 people (52.7\%) reporting that they were able to sneeze into a tissue and then dispose of it only sometimes or almost never. Rates of social distancing were lower still, with 100 (77.5\%) people reporting that they had only sometimes or almost never been able to keep a distance of a metre when infected or from infected people. Only 7 respondents (5.4\%) had ever worn a mask to prevent infection.

\section{INSERT Table IV}

After controlling for past behaviour, the TPB behavioural determinants did explain further variance in intentions for each behaviour (Table IV), but the combination of determinants differed for each behaviour. Instrumental attitude contributed to both handwashing and cough-sneeze hygiene intentions, but not to mask-wearing or social distancing. Perceived behavioural control did not contribute to hand-washing intentions, but did contribute to all other intentions, and was the foremost predictor of cough-sneeze hygiene intentions. Subjective norm contributed to all intentions except mask-wearing, and hand- 
washing was also predicted by descriptive norms. Unusually, affective attitude did not contribute significantly to any of the intention measures after controlling for the other determinants. After controlling for the TPB determinants, one of the measures of flu risk contributed to intentions for every behaviour, and flu risk dominated the prediction of maskwearing.

\section{INSERT Table V}

All of the behavioural and control beliefs we assessed were significantly related to hand-washing intentions and current behaviour (Table V), with mainly moderate to strong associations. The strongest predictor of intention and behaviour was the negative affective belief that frequent hand-washing would feel like over-reacting. The positive instrumental belief that hand-washing would protect others was the strongest predictor of intentions. Control beliefs relating to feeling that hand-washing would be hard to remember and carry out when busy were moderately negatively correlated with intentions but strongly correlated with behaviour.

\section{Discussion}

The questionnaire results confirmed our key assumption, that hand-washing would be viewed as the most feasible preventive behaviour. Although cough-sneeze hygiene was the behaviour viewed most favourably, perceptions of control over being able to perform this behaviour were low, and most people reported seldom being able to successfully ‘catch’ and 'bin' coughs and sneezes (as recommended by the UK government campaign). In order to avoid the risk of fostering fatalistic and sceptical attitudes towards preventive measures it is important that health promotion interventions target behaviours that are regarded as feasible, confirming our selection of hand-washing as the most appropriate principal focus for our web-based intervention. 
The questionnaire study also confirmed that the beliefs identified in the qualitative study were strongly related to hand-washing intentions and current behaviour. Moreover, while intentions were strongly correlated with current behaviour, the regression analyses confirmed that after controlling for current behaviour a substantial proportion of the variance in intentions to carry out each behaviour in the future was explained by the TPB behavioural determinants, with some additional contribution from descriptive norms and perceived flu risk. These findings provided useful confirmation that our intervention appeared to be addressing issues related to intentions to increase hand-washing frequency.

The qualitative studies revealed additional beliefs that could influence hand-washing that led us to make significant modifications to our website intervention. Our target behaviour was changed to hand-washing 10 times a day, since most users believed that they were already meeting the lower target of 6 times a day, and the higher rate was compatible with expert advice for the pandemic. A quiz was introduced to allow users to check their knowledge of how viruses are transmitted, in order to identify and challenge misleading beliefs (e.g. that catching minor infections is beneficial in order to strengthen the immune system). Webpages were added to advise on how to avoid dry hands, and to reassure users that prudent hand-washing was socially sanctioned and not obsessive.

A potentially important finding from the quantitative study was the unusually strong association of social norms with these behaviours; typically, norms are less relevant than attitudes and perceived control (Godin \& Kok, 1996). This finding is consistent with the evidence from qualitative studies, including ours, that hygienic behaviour is largely governed by socio-cultural customs and motivated by social approval (Curtis et al., 2009; Whitby et al., 2006). Our website attempted to address social influences on hand-washing by analogy with other normative and socially valued practices (e.g. cleaning teeth, using deodorant) and encouraging involvement of all household members in the intervention. However, campaigns 
to directly change social norms and practices at a population level might prove useful, such as ensuring that role models adopt and demonstrate hygienic behaviours, and providing facilities such as hand-gel dispensers in public places (e.g. where food is eaten) to both facilitate and endorse their habitual public use (Curtis et al., 2009).

\section{Limitations and conclusion}

A limitation of these studies is that they relied on self-report. Observed levels of infection control behaviour tend to be lower than self-reported rates (Lau et al., 2007; Judah, Aunger, Schmidt, Michie, Granger \& Curtis, 2009), and so there may be greater scope for increasing hand-washing levels than might be inferred from the relatively high levels of self-reported hand-washing found in our study. A related issue is that our sample may have been unusually motivated to undertake preventive behaviour as they were a self-selected sub-group of those invited to assist us with developing the intervention. However, the hand-washing rates they reported were actually slightly lower than those described in population studies carried out during the pandemic (Rubin, Amlot, Page \& Wessely, 2009; Yuan, Zhang, Xu, Shen, Zhange \& Ma, 2009).

While these studies were able to identify explicit motivations, behaviour is also influenced by non-rational and automatic processes that are less easy to report, such as habit (Oulette \& Wood, 1998). The finding that control beliefs predicted hand-washing behaviour more strongly than hand-washing intentions suggests that respondents are aware that forgetting and environmental circumstances may prevent them from carrying out intended behaviour.

In conclusion, by using mixed methods we were able to confirm the assumptions on which our intervention development was based, and particularly our choice of hand-washing as the most feasible behaviour to target. We were also able to identify important additional 
beliefs and factors that should be addressed by interventions aiming to promote behaviour to reduce infection transmission. Although the expected associations between intentions and behavioural determinants were observed in these studies, to demonstrate a causal link it will be necessary to show that our intervention is able to influence intentions and behaviour by changing the relevant beliefs and attitudes. 


\section{Acknowledgements}

We would like to thank Leanne Morrison for carrying out some of the think aloud sessions and Adrian Osmond for support for the website development.

The web-based materials and survey used for this study were created using the LifeGuide system developed as part of the national Digital Social Science programme funded by the UK Economic and Social Research Council.

\section{Funding}

This work was supported by the UK Medical Research Council [grant number GO700459]. 


\section{Reference List}

Ajzen, I. (1991). The theory of planned behavior. Organizational Behaviour and Human Decision Processes, 50(2), 179-211.

Ajzen, I. (2002). Perceived behavioral control, self-efficacy, locus of control, and the theory of planned behavior. Journal of Applied Social Psychology, 32, 1-20.

Bell, D., Nicoll, A., Fukuda, K., Horby, P., Monto, A., Hayden, F., et al. (2006) Nonpharmaceutical interventions for pandemic influenza, national and community measures. Emerging Infectious Diseases, 12(1), 88-94.

Bourgeois, F.T., Simons, W.W., Olson, K., Brownstein, J.S., \& Mandl, K.D. (2008). Evaluation of influenza prevention in the workplace using a personally controlled health record: Randomized controlled trial. Journal of Medical Internet Research, 10(1).

Braun, V., \& Clarke, V. (2006). Using thematic analysis in psychology. Qualitative Research in Psychology, 3, 77-101.

Brewer, N.T., Chapman, G.B., Gibbons, F.X., Gerrard, M., McCaul, K.D., \& Weinstein, N.D. (2007). Meta-analysis of the relationship between risk perception and health behaviour: the example of vaccination. Health Psychology, 26, 136-145.

Cava, M.A., Fay, K.E., Beanlands, H.J., McCay, E.A., \& Wignall, R. (2005). Risk perception and compliance with quarantine during the SARS outbreak. Journal of Nursing Scholarship, 37(4), 343-347.

Cotton, D., \& Gresty, K. (2006). Reflecting on the think-aloud method for evaluating elearning. British Journal of Educational Technology, 37(1), 45-54. 
Cowling, B.J., Chan, K.H., Fang, V.J., Cheng, C.K.Y., Fung, R.O.P., Wai, W., et al. (2009). Facemasks and Hand Hygiene to Prevent Influenza Transmission in Households A Cluster Randomized Trial. Annals of Internal Medicine, 151(7), 437-W144.

Craig, P., Dieppe, P., Macintyre, S., Michie, S., Nazareth, I., \& Petticrew, M. (2008) Developing and evaluating complex interventions: the new Medical Research Council guidance. British Medical Journal, 337, a1655.

Curtis, V.A., Danquah, L.O., \& Aunger, R.V. (2009). Planned, motivated and habitual hygiene behaviour: an eleven country review. Health Education Research, 24, 655673.

Gittelsohn, J., Steckler, A., Johnson, C.C., Pratt, C., Grieser, M., Piclcrel, J., et al. (2006). Formative research in school and community-based health programs and studies: "State of the art" and the TAAG approach. Health Education \& Behaviour, 33(1), 2539.

Godin, G., \& Kok, G. (1996). The theory of planned behavior: a review of its applications to health-related behaviors. American Journal of Health Promotion, 11, 87-98.

Green, L.W., \& Kreuter, M.W. (1999). Health Promotion Planning: An Educational and Ecological Approach. Mountain View, CA: Mayfield.

Jefferson, T., Foxlee, R., Del Mar, C., Dooley, L., Ferroni, E., Hewak, B., et al. (2008). Physical interventions to interrupt or reduce the spread of respiratory viruses: systematic review. British Medical Journal, 336(7635), 77-80.

Joffe, H., \& Yardley, L. (2004). Content and thematic analysis. In: D. Marks, \& L. Yardley, (Eds.), Research Methods for Clinical and Health Psychology (pp. 56-68). London: Sage. 
Judah, G., Aunger, R., Schmidt, W.P., Michie, S., Granger, S., \& Curtis, V. (2009). Experimental Pretesting of Hand-Washing Interventions in a Natural Setting. American Journal of Public Health, 99, S405-S411.

Lau, J.T.F., Kim, J.H., Tsui, H.Y., \& Griffiths, S. (2007). Anticipated and current preventive behaviors in response to an anticipated human-to-human H5N1 epidemic in the Hong Kong Chinese general population. BioMed Central Infectious Diseases, 7, 18.

Lau, J.T.F., Yang, X., Tsui, H., \& Kim, J.H. (2003). Monitoring community responses to the SARS epidemic in Hong Kong: from day 10 to day 62. Journal of Epidemiology \& Community Health, 57(11), 864-870.

Leaker, D. (2008). Sickness absence from work in the UK. Economic and Labour Market Review, 2, 18-22.

Leppin, A., \& Aro, A.R. (2009). Risk perceptions related to SARS and avian influenza: theoretical foundations of current empirical research. International Journal of Behavioural Medicine, 16, 7-29.

Leung, G.H., Quah, S., Ho, L.M., Hedley, A.J., Lee, H.P., \& Lam, T.H. (2004). A tale of two cities: community psychobehavioral surveillance and related impact on outbreak control in Hong Kong and Singapore during the severe acute respiratory syndrome epidemic. Infection Control \& Hospital Epidemiology, 25, 1033-1041.

Leung, G.M., Ho, L.-M., Chan, S.K.K., Ho, S.-Y., Bacon-Shone, J., Choy, R.Y.L., et al. (2005). Longitudinal assessment of community psychobehavioral responses during and after the 2003 outbreak of severe acute respiratory syndrome in Hong Kong. Clinical Infectious Diseases, 40(12), 1713-1720.

Maclntyre, C.R., Cauchemez, S., Dwyer, D.E., Seale, H., Cheung, P., Browne, G., et al. (2009). Face Mask Use and Control of Respiratory Virus Transmission in Households. Emerging Infectious Diseases, 15(2), 233-241. 
Madjid, M., Naghavi, M., Litovsky, S., \& Casscells, S.W. (2003). Influenza and cardiovascular disease - A new opportunity for prevention and the need for further studies. Circulation, 108(22), 2730-2736.

Meier, C.R., Jick, S.S., Derby, L.E., Vasilakis, C., \& Jick, H. Acute respiratory-tract infections and risk of first-time acute myocardial infarction. Lancet, 351(9114), 14671471.

Morrison, L., \& Yardley, L. (2009). What infection control measures will people carry out to reduce transmission of pandemic influenza? A focus group study. BioMed Central Public Health, 9, 258.

Oulette, J.A., \& Wood, W. (1998). Habit and intention in everyday life: the multiple processes by which past behavior predicts future behavior. Psychological Bulletin, 124, 54-74.

Rabie, T., \& Curtis, V. (2006). Handwashing and risk of respiratory infections: A quantitative systematic review. Tropical Medicine \& International Health, 11(3), 258-267.

Rhodes, R.E., Blanchard, C.M., \& Matheson, D.H. (2006). A multicomponent model of the theory of planned behaviour. British Journal of Health Psychology, 11, 119-137.

Rivis, A., \& Sheeran, P. (2003). Descriptive norms as an additional predictor in the Theory of Planned Behaviour: a meta-analysis. Current Psychology, 22, 218-233.

Rubin, G.J., Amlot, R., Page, L., \& Wessely, S. (2009). Public perceptions, anxiety, and behaviour change in relation to the swine flu outbreak: cross sectional telephone survey. British Medical Journal, 339(jul02_3), b2651.

Slaughter, L., Keselman, A., Kushniruk, A., Patel, V.L. (2005). A framework for capturing the interactions between laypersons' understanding of disease, information gathering behaviors, and actions taken during an epidemic. Journal of Biomedical Informatics, 38(4), 298-313. 
Sutton, S. (2002). Using social cognition models to develop health behaviour interventions: problems and assumptions. In: D. Rutter, \& L. Quine (Eds.), Changing health behaviour (pp. 193-208). Buckingham: Open University Press.

Tang, C.S.-K., \& Wong, C.-Y. (2004) Factors influencing the wearing of facemasks to prevent the severe acute respiratory syndrome among adult Chinese in Hong Kong. Preventative Medicine, 39(6), 1187-1193.

van den Haak, M.J., de Jong, M.D.T., \& Schellens, P.J. (2007). Evaluation of an informational web site: Three variants of the think-aloud method compared. Technical Communications, 54, 58-71.

Webb, T.L., \& Sheeran, P. (2006). Does changing behavioral intentions engender behavior change? A meta-analysis of the experimental evidence. Psychological Bulletin, 132, 249-268.

Whitby, M., McLaws, M.L., \& Ross, M.W. (2006). Why healthcare workers don't wash their hands: a behavioral explanation. Infection Control \& Hospital Epidemiology, 27, 484492.

Woodhead, M.A., Macfarlane, J.T., Mccracken, J.S., Rose, D.H., \& Finch, R.G. (1987). Prospective-Study of the Etiology and Outcome of Pneumonia in the Community. Lancet, 1(8534), 671-674.

Yuan, J., Zhang, L., Xu, W., Shen, J., Zhange, P., \& Ma, H. (2009). Reported changes in health-related behaviours in Chinese urban residents in response to an influenza pandemic. Epidemiology \& Infection, 137, 988-993. 